\title{
Testing for Common Deterministic Trend Slopes
}

\author{
Timothy J. Vogelsang* \\ Department of Economics \\ Cornell University \\ Philip Hans Franses \\ Econometric Institute \\ Erasmus University Rotterdam
}

June 13, 2001

\begin{abstract}
We propose tests for hypotheses on the parameters for deterministic trends. The model framework assumes a multivariate structure for trend-stationary time series variables. We derive the asymptotic theory and provide some relevant critical values. Monte Carlo simulations suggest which tests are more useful in practice than others. We apply our tests to examine if monthly temperatures in The Netherlands, measured from 1706 onwards, have a trend and if these trends are the same across months. We find that the January and March temperatures have the same upward trend, that the September temperature has decreased and that the temperatures in the other months do not have a trend. Hence, only winters in The Netherlands seem to get warmer.
\end{abstract}

Key words: Deterministic trends, Hypothesis testing, Monthly temperatures, Multivariate Trend Function Testing

\footnotetext{
*Address for correspondence: T.J. Vogelsang, Department of Economics, Uris Hall, Cornell University, Ithaca, NY 14853-7601, e-mail: tjv2@cornell.edu. The computer programs used for all calculations in this paper can be obtained from the corresponding author. Additional simulation results can also be obtained upon request. Work on this paper was initiated when the first author visited the Tinbergen Institute Rotterdam (1998) and the second author visited Cornell University (May 1999).
} 


\section{Introduction}

In several empirical situations it is found that time series data contain a deterministic trend, while they are otherwise stationary. An example in macroeconomics concerns differences between real output for pairs of countries (see Hobijn and Franses (2000) among others) or pairs of regions within the U.S. (see Carlino and Mills (1993), Loewy and Papell (1995) and Tomljanovich and Vogelsang (2001) among others), where such a trend-stationary series indicates some degree of convergence. Other examples can be found in disciplines such as tourism and marketing, where tourist arrivals and sales often display upward trending patterns. Finally, environmental data like temperatures may also display trends, and if these are upward moving this can be taken as evidence of global warming. See Bloomfield (1992), Woodward and Gray (1993), Zheng and Basher (1999) and Fomby and Vogelsang (2000) among many others.

In some of the above cases it may be of interest to examine if two or more trend-stationary time series have the same slope. This would allow for testing whether a pair of countries are converging with the same speed as another pair. Also, one may want to test whether temperatures across countries have the same upward moving trend. In this paper we propose tests for such and related hypotheses. We should note that while there has been recent research on univariate trend function inference and modeling (see Perron (1991), Canjels and Watson (1997), Vogelsang $(1997,1998)$ ), multivariate trend modeling and inference has received little attention ${ }^{1}$.

The outline of our paper is as follows. In Section 2, we discuss the model representation, parameter estimation, and the test statistics of interest. A key issue is the estimation of the covariance matrix, for which we aim to compare three different approaches, amongst which the familiar HAC estimator. In Section 3, we derive the relevant asymptotic theory. We tabulate useful critical values. Additionally, we discuss asymptotic power of the tests in a special case. In Section 4, we rely on Monte Carlo simulations to examine the finite sample performance of the test statistics. We observe that the tests work best for a limited number of variables under scrutiny. Additionally, we find that the HAC-based tests have serious size distortions, while our new tests perform satisfactorily. In Section 5, we apply our tests to three centuries of monthly temperatures for The Netherlands. We find that the January and March temperatures have the same upward

\footnotetext{
${ }^{1}$ It should be noted that the asymptotic theory for multivariate time series regressions developed by Park and Phillips (1988) includes our model as a special case where no covariates are included. However, they do not consider hypotheses involving cross equation restrictions on the linear trend parameters as we do here.
} 
trend, that the September temperature has decreased over time and that the temperatures in the other months do not have a trend. Hence, only winters in The Netherlands seem to get warmer.

\section{The model and test statistics}

In this section we present the model, parameter estimation and the relevant test statistics.

\subsection{Representation}

Consider $n$ trend-stationary time series denoted by $y_{1, t}$ to $y_{n, t}$ with $t=1,2, \ldots, T$, and assume that they can be represented by

$$
\begin{gathered}
y_{1, t}=\mu_{1}+\beta_{1} t+u_{1, t} \\
y_{2, t}=\mu_{2}+\beta_{2} t+u_{2, t} \\
\cdots \\
y_{n, t}=\mu_{n}+\beta_{n} t+u_{n, t} .
\end{gathered}
$$

Define the three $n \times 1$ vectors $u_{t}, \mu$ and $\beta$ by $\left(u_{1, t}, u_{2, t}, \ldots, u_{n, t}\right)^{\prime},\left(\mu_{1}, \mu_{2}, \ldots, \mu_{n}\right)^{\prime}$ and $\left(\beta_{1}, \beta_{2}, \ldots, \beta_{n}\right)^{\prime}$, respectively. It is assumed that a functional limit theorem applies to $u_{t}$, that is,

$$
T^{-\frac{1}{2}} \sum_{t=1}^{[r T]} u_{t} \Rightarrow \Lambda W_{n}(r)
$$

where $\Rightarrow$ denotes weak convergence, $W_{n}(r)$ is an $n \times 1$ standard independent Wiener process, and $[r T]$ is the integer part of $r T$. See, for example, Phillips and Durlauf (1986) for conditions under which (2) holds. We denote $\Omega$ as the long-run variance of $u_{t}$, that is,

$$
\Omega=\Lambda \Lambda^{\prime}=\sum_{j=-\infty}^{\infty} \Gamma_{j},
$$

where $\Gamma_{j}=\operatorname{Cov}\left[u_{t} u_{t-j}^{\prime}\right]$. The hypotheses of interest in this paper are

$$
\begin{aligned}
& H_{0}: R \beta=r \\
& H_{1}: R \beta \neq r,
\end{aligned}
$$

where $R$ is a $q \times n$ matrix, and $r$ is a $n \times 1$ vector. 


\subsection{Estimation}

The parameters in (1) can be estimated by applying ordinary least squares (OLS) equation by equation, which results in $\widehat{\mu}$ and $\widehat{\beta}$. If the errors are second order stationary (a typical condition under which (2) will hold), then from the classic results of Grenander and Rosenblatt (1957), OLS is asymptotically equivalent to GLS (and MLE under Gaussian errors). In addition, because (1) is a seemingly unrelated regression (SUR) with the same regressors in each equation, OLS is equivalent to the SUR estimator, which is the GLS estimator for i.i.d. errors with different error variances. Thus, OLS has some nice optimality properties.

It will be convenient to express $\widehat{\beta}_{i}$ as follows. Define $\bar{t}=T^{-1} \sum_{t=1}^{T} t$ and $\tilde{t}=t-\bar{t}$, then

$$
\widehat{\beta}_{i}=\left(\sum_{t=1}^{T} \widetilde{t}^{2}\right)^{-1} \sum_{t=1}^{T} \widetilde{t} y_{i, t}
$$

for $i=1,2, \ldots, n$. These estimators can be summarized into the $n \times 1$ vector $\widehat{\beta}=\left(\widehat{\beta}_{1}, \widehat{\beta}_{2}, \ldots, \widehat{\beta}_{n}\right)^{\prime}$. As usual we obtain

$$
\widehat{\beta}-\beta=\left(\sum_{t=1}^{T} \widetilde{t}^{2}\right)^{-1} \sum_{t=1}^{T} \widetilde{t} u_{t}
$$

To derive the asymptotic distribution of (4), note that

$$
T^{-3} \sum_{t=1}^{T} \widetilde{t}^{2} \rightarrow \frac{1}{12}
$$

where $\rightarrow$ denotes convergence, and that

$$
T^{-\frac{3}{2}} \sum_{t=1}^{T} \widetilde{t} u_{t} \Rightarrow \Lambda \int_{0}^{1}\left(r-\frac{1}{2}\right) d W_{n}(r)=\Lambda\left[\frac{1}{2} W_{n}(1)-\int_{0}^{1} W_{n}(r) d r\right] .
$$

For later it will be useful to define the process

$$
V_{n}(r)=\left(r-\frac{1}{2}\right) W_{n}(r)-\int_{0}^{r} W_{n}(s) d s-W_{n}(1) \int_{0}^{r}\left(s-\frac{1}{2}\right) d s,
$$

where

$$
V_{n}(1)=\left[\frac{1}{2} W_{n}(1)-\int_{0}^{1} W_{n}(r) d r\right]
$$


follows from the fact that $\int_{0}^{1}\left(s-\frac{1}{2}\right) d s=0$. Using (5), (6) and (2.2), we obtain

$$
T^{\frac{3}{2}}(\widehat{\beta}-\beta)=\left(T^{-3} \sum_{t=1}^{T} \widetilde{t}^{2}\right)^{-1} T^{-3 / 2} \sum_{t=1}^{T} \widetilde{t} u_{t} \Rightarrow 12 \Lambda V_{n}(1)
$$

It is easy to show that $12 \Lambda V_{n}(1)$ is distributed as $N(0,12 \Omega)$. Therefore, inference regarding $\beta$ can be carried out in the usual way provided a consistent estimator of $\Omega$ is available. Under regularity conditions similar to those required for (2) to hold, $\Omega$ can be consistently estimated using the class of heteroskedasticity and autocorrelation consistent (HAC) covariance matrix estimators, see Andrews (1991). Here we focus on the Bartlett kernel (see Newey and West (1987)) estimator defined as

$$
\widehat{\Omega}_{H A C}=\widehat{\Gamma}_{0}+\sum_{j=1}^{T-1}\left(1-\frac{j}{S_{T}}\right)\left(\widehat{\Gamma}_{j}+\widehat{\Gamma}_{j}^{\prime}\right),
$$

where $\widehat{\Gamma}_{j}=T^{-1} \sum_{t=j+1}^{T} \widehat{u}_{t} \widehat{u}_{t-j}^{\prime}$ and $S_{T}$ is the truncation lag or bandwidth. For $\widehat{\Omega}_{H A C}$ to be consistent, $S_{T}$ must increase as the sample increases but at a slower rate. Andrews (1991) showed that the rate $T^{\frac{1}{3}}$ minimizes the approximate MSE for $\widehat{\Omega}$. In the Monte Carlo simulations and empirical work that follows, we choose $S_{T}$ using the data-dependent method suggested by Andrews (1991) based on the VAR(1) plug-in formula.

We now consider alternatives to using consistent estimates of $\widehat{\Omega}$. Following Kiefer, Vogelsang and Bunzel (2000) and Kiefer and Vogelsang (2000), suppose we set $S_{T}=T$ in (10). Let $\widehat{\Omega}_{S_{T}=T}$ denote this estimator of $\Omega$. Although $S_{T}=T$ does not result in a consistent estimator of $\Omega$, valid testing is still possible because $\widehat{\Omega}_{S_{T}=T}$ is asymptotically proportional to $\Omega$ as is shown below. The advantage of $\widehat{\Omega}_{S_{T}=T}$ is that it uses a specific bandwidth and this choice of bandwidth is captured by the first-order asymptotics.

The asymptotic behavior of $\widehat{\Omega}_{S_{T}=T}$ is as follows. Following Kiefer and Vogelsang (2001) and using the fact that $\sum_{j=1}^{T} \widehat{u}_{t}=0$, it holds that

$$
\widehat{\Omega}_{S_{T}=T}=\widehat{\Gamma}_{0}+\sum_{j=1}^{T-1}\left(1-\frac{j}{T}\right)\left(\widehat{\Gamma}_{j}+\widehat{\Gamma}_{j}^{\prime}\right)=2 T^{-2} \sum_{t=1}^{T} \widehat{S}_{t} \widehat{S}_{t}^{\prime},
$$

where $\widehat{S}_{t}=\sum_{j=1}^{t} \widehat{u}_{j}$. In the appendix we prove that

$$
T^{-\frac{1}{2}} \widehat{S}_{[r T]} \Rightarrow \Lambda \widehat{V}_{n}(r)
$$


where $\widehat{V}_{n}(r)=W_{n}(r)-r W_{n}(1)-12 V_{n}(1) \int_{0}^{r}\left(s-\frac{1}{2}\right) d s$. It directly follows from (11), (12) and the continuous mapping theorem that

$$
\widehat{\Omega}_{S_{T}=T}=2 T^{-1} \sum_{t=1}^{T} T^{-1 / 2} \widehat{S}_{t} T^{-1 / 2} \widehat{S}_{t}^{\prime} \Rightarrow 2 \Lambda\left(\int_{0}^{1} \widehat{V}_{n}(r) \widehat{V}_{n}(r)^{\prime} d r\right) \Lambda^{\prime} .
$$

We also consider an alternative to $\widehat{\Omega}_{S_{T}=T}$ which is constructed using $\widetilde{t}_{t}$ instead of $\widehat{u}_{t}$. In a standard application of HAC estimators to the regressions given by $((1)), \widetilde{t} \widehat{u}_{t}$ would be used. Because $\widetilde{t}_{t}$ is not a vector of stationary time series, establishing consistency of a HAC estimator in this case would be difficult if even feasible. However, if we use $S_{T}=T$, the asymptotic behavior of the HAC estimator can be derived. Specifically, define

$$
\widetilde{\Omega}_{S_{T}=T}=\widetilde{\Gamma}_{0}+\sum_{j=1}^{T-1}\left(1-\frac{j}{T}\right)\left(\widetilde{\Gamma}_{j}+\widetilde{\Gamma}_{j}^{\prime}\right)
$$

where

$$
\widetilde{\Gamma}_{j}=T^{-1} \sum_{t=j+1}^{T}\left[(t-\bar{t}) \widehat{u}_{t}\right]\left[(t-j-\bar{t}) \widehat{u}_{t-j}^{\prime}\right] .
$$

Again, using Kiefer and Vogelsang (2001), we can write

$$
\widetilde{\Omega}_{S_{T}=T}=2 T^{-2} \sum_{t=1}^{T} \widetilde{S}_{t} \widetilde{S}_{t}^{\prime}
$$

where $\widetilde{S}_{t}=\sum_{j=1}^{t}(j-\bar{t}) \widehat{u}_{j}$. In the appendix we prove that

$$
T^{-\frac{3}{2}} \widetilde{S}_{[r T]} \Rightarrow \Lambda \widetilde{V}_{n}(r)
$$

where $\widetilde{V}_{n}(r)=V_{n}(r)-12 V_{n}(1) \int_{0}^{r}\left(s-\frac{1}{2}\right)^{2} d s$. It directly follows from (16), (17) and the continuous mapping theorem that

$$
T^{-2} \widetilde{\Omega}_{S_{T}=T}=2 T^{-1} \sum_{t=1}^{T} T^{-3 / 2} \widetilde{S}_{t} T^{-3 / 2} \widetilde{S}_{t}^{\prime} \Rightarrow 2 \Lambda\left(\int_{0}^{1} \widetilde{V}_{n}(r) \widetilde{V}_{n}(r)^{\prime} d r\right) \Lambda^{\prime}
$$

\subsection{Test statistics}

To test the null hypothesis in (3) against the relevant alternative hypothesis, we consider three joint tests for the restrictions in $R$. The first two tests are $F$-tests which we compute using the 
Bartlett HAC estimator with bandwidth $T$. These tests rely either on $\widetilde{\Omega}_{S_{T}=T}$ or on $\widehat{\Omega}_{S_{T}=T}$. The first test statistic is

$$
F_{1}^{*}=T(R \widehat{\beta}-r)^{\prime}\left[R\left(T^{-1} \sum_{t=1}^{T} \widetilde{t}^{2}\right)^{-1} \widetilde{\Omega}_{S_{T}=T}\left(T^{-1} \sum_{t=1}^{T} \widetilde{t}^{2}\right)^{-1} R^{\prime}\right]^{-1}(R \widehat{\beta}-r) / q,
$$

where $\widetilde{\Omega}_{S_{T}=T}$ is defined in (16). The second $F$-test we consider is

$$
F_{2}^{*}=(R \widehat{\beta}-r)^{\prime}\left[R\left(\sum_{t=1}^{T} \widetilde{t}^{2}\right)^{-1} \widehat{\Omega}_{S_{T}=T} R^{\prime}\right]^{-1}(R \widehat{\beta}-r) / q
$$

where $\widehat{\Omega}_{S_{T}=T}$ is defined in (11).

Naturally, if there is only a single restriction to test, that is $q=1$, then one can consider a $t$-test. In the present notation, these tests are

$$
t_{1}^{*}=\frac{T^{\frac{1}{2}}(R \widehat{\beta}-r)}{\left[R\left(T^{-1} \sum_{t=1}^{T} \widetilde{t}^{2}\right)^{-1} \widetilde{\Omega}_{S_{T}=T}\left(T^{-1} \sum_{t=1}^{T} \widetilde{t}^{2}\right)^{-1} R^{\prime}\right]^{\frac{1}{2}}}
$$

and

$$
t_{2}^{*}=\frac{(R \widehat{\beta}-r)}{\left[R\left(\sum_{t=1}^{T}{\widetilde{t^{2}}}^{2}-1 \widehat{\Omega}_{S_{T}=T} R^{\prime}\right]^{\frac{1}{2}}\right.},
$$

respectively.

The standard alternative to $F_{1}^{*}$ and $F_{2}^{*}$ is a Wald test based on $\widehat{\Omega}_{H A C}$ defined as

$$
W_{H A C}=(R \widehat{\beta}-r)^{\prime}\left[R\left(\sum_{t=1}^{T} \widetilde{t}^{2}\right)^{-1} \widehat{\Omega}_{H A C} R^{\prime}\right]^{-1}(R \widehat{\beta}-r) .
$$

Likewise, we defined the standard HAC $t$-test as

$$
t_{H A C}=\frac{(R \widehat{\beta}-r)}{\left[R\left(\sum_{t=1}^{T} \widetilde{t}^{2}\right)^{-1} \widehat{\Omega}_{H A C} R^{\prime}\right]^{\frac{1}{2}}} .
$$

\section{Asymptotic theory and critical values}

In this section we develop the asymptotic theory for the tests. We also compute useful critical values. Finally, we discuss asymptotic power. 


\subsection{Asymptotic distributions}

Assumption (2) is sufficient to obtain the asymptotic null distributions of $F_{1}^{*}$ and $F_{2}^{*}$. The asymptotic distributions of these tests are summarized in the following Theorem and Corollary.

Theorem 1 Let the data be generated by (1) and suppose assumption (2) holds. Then, as $T \rightarrow \infty$,

$$
\begin{aligned}
& F_{1}^{*} \Rightarrow V_{q}(1)^{\prime}\left[2 \int_{0}^{1} \widetilde{V}_{q}(r) \widetilde{V}_{q}(r)^{\prime} d r\right]^{-1} V_{q}(1) / q, \\
& F_{2}^{*} \Rightarrow V_{q}(1)^{\prime}\left[\frac{1}{6} \int_{0}^{1} \widehat{V}_{q}(r) \widehat{V}_{q}(r)^{\prime} d r\right]^{-1} V_{q}(1) / q .
\end{aligned}
$$

Corollary 1 Let the data be generated by (1) and suppose assumption (2) holds. If $q=1$, then as $T \rightarrow \infty$,

$$
\begin{aligned}
& t_{1}^{*} \Rightarrow \frac{V_{1}(1)}{\left(2 \int_{0}^{1} \widetilde{V}_{1}(r)^{2} d r\right)^{\frac{1}{2}}}, \\
& t_{2}^{*} \Rightarrow \frac{V_{1}(1)}{\left(\frac{1}{6} \int_{0}^{1} \widehat{V}_{1}(r)^{2} d r\right)^{\frac{1}{2}}} .
\end{aligned}
$$

Note that $F_{1}^{*}$ and $F_{2}^{*}$ are quadratic forms involving the normal random vector $V_{q}(1)$ and random matrices that reflect the use of $\widetilde{\Omega}_{S_{T}=T}$ and $\widehat{\Omega}_{S_{T}=T}$.

Finally, if $\widehat{\Omega}_{H A C}$ is a consistent estimator of $\Omega$, then the asymptotic distribution of the Wald test $W_{H A C}$ is $\chi^{2}$ with $q$ degrees of freedom. When $q=1, t$-tests based on $\widehat{\Omega}_{H A C}$ are asymptotically distributed as $N(0,1)$.

\subsection{Critical values}

The critical values for the $t_{1}^{*}, t_{2}^{*}, F_{1}^{*}$ and $F_{2}^{*}$ need to be obtained through Monte Carlo simulation. The asymptotic critical values were simulated using 50,000 replications. The Wiener processes were approximated by normalized sums of i.i.d $N(0,1)$ errors using 1,000 steps. The critical values for the $t_{1}^{*}$ and $t_{2}^{*}$ tests are given in Table 1 . Right tail critical values are given. The left tail critical 
values follow from symmetry around zero. The critical values for the $F_{1}^{*}$ test are given in Table 2 , where we tabulate the critical values for tests for $q$ restrictions, where $q$ runs from 1 to 30. Similarly obtained critical values for the $F_{2}^{*}$ test are given in Table 3 .

\subsection{Asymptotic power}

One way to compare and contrast the new tests with each other and with standard HAC-based tests is to examine asymptotic power for local alternatives. To keep the analysis transparent, we consider the case of $q=1$ and focus on the regression

$$
y_{1, t}=\mu_{1}+\beta_{1} t+u_{1, t}
$$

Let $\sigma_{1}^{2}=\gamma_{0}+\sum_{j=-\infty}^{\infty} \gamma_{j}$, where $\gamma_{j}=\operatorname{Cov}\left(u_{1, t}, u_{1, t-j}\right)$. Then, under assumption (2),

$$
T^{-\frac{1}{2}} \sum_{t=1}^{[r T]} \Rightarrow \sigma_{1} W_{1}(r) .
$$

Consider the one-sided hypothesis $H_{0}: \beta_{1} \leq \beta_{0}$ against $H_{1}: \beta_{1}>\beta_{0}$. We can obtain nondegenerate limiting distributions for the $t$-tests under the local alternative $\beta_{1}=\beta_{0}+c T^{-\frac{3}{2}}$. Thus, $\beta_{1}$ converges to $\beta_{0}$ at rate $T^{-\frac{3}{2}}$.

The following theorem gives the limiting distribution of the tests under the local alternative. The proof is given in the appendix.

Theorem 2 Suppose the data are generated by (19) and that assumption (2) holds. Suppose $t-t e s t s$ are constructed for testing $H_{0}: \beta_{1} \leq \beta_{0}$ against $H_{1}: \beta_{1}>\beta_{0}$. Suppose $\beta_{1}=\beta_{0}+c T^{-\frac{3}{2}}$, then, as $T \rightarrow \infty$,

$$
\begin{gathered}
t_{H A C} \Rightarrow \frac{\delta+12 V_{1}(1)}{\sqrt{12}}, \\
t_{1}^{*} \Rightarrow \frac{\delta+12 V_{1}(1)}{\left(288 \int_{0}^{1} \widetilde{V}_{1}^{2}(r) d r\right)^{1 / 2}}, \\
t_{2}^{*} \Rightarrow \frac{\delta+12 V_{1}(1)}{\left(24 \int_{0}^{1} \widehat{V}_{1}^{2}(r) d r\right)^{1 / 2}},
\end{gathered}
$$

where $\delta=c / \sigma_{1}$. 
Using Theorem 3, asymptotic power can be computed by simulating the distributions under the local alternative for various values of $\delta$ and computing rejection probabilities using the asymptotic null critical values. Using the same simulation methods used to compute null critical values, we computed asymptotic power for $\delta \in[0,20]$. Power with a nominal level of $5 \%$ is plotted in Figure 1. Clearly, power is highest for $t_{H A C}$, followed by $t_{2}^{*}$ and then $t_{1}^{*}$. The reason that $t_{1}^{*}$ and $t_{2}^{*}$ have slightly lower power is because they use "standard errors" with sampling variability that does not vanish as $T$ increases. However, as we will show in the next section, $t_{H A C}$ is generally more size distorted in finite samples. Thus, $t_{1}^{*}$ and $t_{2}^{*}$ trade off power in exchange for better size.

\section{Finite sample performance}

In this section we examine the finite sample performance of the $F_{1}^{*}$ and $F_{2}^{*}$ tests and compare them to $W_{H A C}$. We generate data according to (1) with $n=12$ (to match the empirical application) where the errors are modeled as $\mathrm{AR}(1)$ processes, that is

$$
u_{i, t}=\rho u_{i, t-1}+e_{i, t},
$$

with $e_{i, t}$ i.i.d. $N(0,1)$ and $u_{i, 0}=0$. For the sake of simplicity, we use the same $\operatorname{AR}(1)$ coefficient for all series. We consider the following null hypotheses:

$$
\begin{aligned}
& H_{0}^{1}: \beta_{1}=0, \\
& H_{0}^{2}: \beta_{1}=\beta_{2}=0, \\
& H_{0}^{3}: \beta_{1}=\beta_{2}=\beta_{3}=0, \\
& H_{0}^{4}: \beta_{1}=\beta_{2}=\ldots=\beta_{11}=0,
\end{aligned}
$$

for $q=1,2,3,11$, respectively. To explore the finite sample size of the tests, we generate data under these null hypotheses and we set the intercepts and other slopes to zero as the three tests are exactly invariant to those parameters. To compare power of the tests, we generate data under the alternatives

$$
\begin{aligned}
& H_{1}^{1}: \beta_{1}=\beta, \\
& H_{1}^{2}: \beta_{1}=\beta_{2}=\beta, \\
& H_{1}^{3}: \beta_{1}=\beta_{2}=\beta_{3}=\beta, \\
& H_{1}^{4}: \beta_{1}=\beta_{2}=\ldots=\beta_{11}=\beta,
\end{aligned}
$$


respectively. The results are summarized in Tables 4 to 7 . We use $T=100$ and 2,000 replications in all cases. We only report results for $\rho=0,0.2,0.4,0.6,0.8$ and $\beta=0,0.05,0.10,0.20$. We implement the tests both with and without VAR(1) prewhitening (see Andrews and Monahan (1992)). Empirical rejection probabilities under the null hypotheses were computed using 5\% asymptotic critical values. Empirical rejection probabilities under the alternatives were computed using $5 \%$ empirical finite sample critical values (obtained from the null distributions). Thus, finite sample power is size corrected so that power comparisons are meaningful.

In Table 4 we give the results for $H_{0}^{1}$. Several patterns emerge. First, in nearly all cases, empirical null rejection probabilities of $F_{1}^{*}$ and $F_{2}^{*}$ are closer to 0.05 than $W_{H A C}$. This is especially true as $\rho$ increases. Second, prewhitening improves the size of all the tests. This result should be taken with a grain of salt as the prewhitening filter exactly matches the autocorrelation structure of the errors, which is an unlikely situation in practice. Third, and as expected given finite sample results in Kiefer et al. (2000) and Kiefer and Vogelsang (2000), the size-corrected power of $W_{H A C}$ is higher than $F_{1}^{*}$ and $F_{2}^{*}$. This higher power comes at the expense of greater size distortion. Fourth, the size of $F_{1}^{*}$ and $F_{2}^{*}$ are very similar while $F_{2}^{*}$ clearly has higher power. This suggests that $F_{2}^{*}$ would be preferred over $F_{1}^{*}$ in practice.

The dominance of $F_{2}^{*}$ over $F_{1}^{*}$ is continued in Tables 5,6 and $7 . F_{2}^{*}$ always has higher power than $F_{1}^{*}$, except when $\rho=0.8$, in which case the empirical null rejection probabilities of the two tests are similar. And, when prewhitening is used, $F_{2}^{*}$ tends to be less size distorted.

Table 6 shows that while $F_{1}^{*}$ and $F_{2}^{*}$ have reasonable size, especially for $\rho \leq 0.6$ when prewhitening is used, $W_{H A C}$ tends to over-reject even for $\rho$ close to zero. All the tests have over-rejection problems for large $q$ as Table 7 shows. $W_{H A C}$ is particularly bad with rejection probability of 0.158 and 0.419 for $\rho=0$ and $\rho=0.2$ (without prewhitening). $F_{1}^{*}$ and $F_{2}^{*}$ are less distorted, but still over-reject. Prewhitening does not help much, especially when $\rho$ is large. $F_{2}^{*}$ clearly dominates $F_{1}^{*}$ when $q=11$.

Finally, as our empirical example in the next section concerns 288 observations, we simulate the size of the three tests for various values of $\rho$. In general, size distortions are less severe for all the tests compared to $T=100$. We only report results for the $q=11$ case in Table 8 . $F_{2}^{*}$ has reasonable size for $\rho \leq 0.6$ when prewhitening is used. $W_{H A C}$, on the other hand, still tends to over reject even for $\rho$ close to zero. 


\section{Monthly trends in Dutch temperatures}

We now use the tests developed in this paper to analyze monthly trends in temperature series for The Netherlands for the years 1706 to 1993 . We obtained monthly averages of temperatures measured in degrees centigrade for The Netherlands from the Royal Netherlands Meteorology Institute. Using this data, we can ask how the apparent increase in global temperatures has impacted temperatures in The Netherlands. In particular, we are interested in learning whether global warming has uniformly affected temperatures in The Netherlands across the months of the year or whether there are seasonally varying patterns.

We disaggregate the data into 12 annual temperature series corresponding to each month of the year. We fit model (1) to the series using OLS. Of direct interest are the point estimates of the $\beta_{i}$ coefficients as they measure the average annual increase of temperature over time. The point estimates are reported in Table 9, together with measures which indicate that there is almost no serial correlation in the residuals ${ }^{2}$. Notice that the point estimates are not uniform over the months. The largest positive trends are in the January, March and December series. Perhaps surprisingly, August and September have negative trends. The remaining months have mostly positive but small trends.

To put the point estimates in perspective, consider the largest $\widehat{\beta}_{i}$ (January) which is 0.0069. This suggests that January temperatures have increased 0.0069 degrees centigrade per year, or 0.69 degrees per 100 years. This is slightly higher that the annual global increase of temperatures that has been consistently estimated in the 0.004 to 0.006 range in the global warming literature ${ }^{3}$. The March point estimate of 0.0038 is closer to the annual global rate.

In terms of statistical evidence, the January and March slopes are statistically larger than zero at the $5 \%$ or $10 \%$ levels, depending on the test used. The September slope is statistically significant and less than zero. All other months have point estimates that are not significantly different from zero. The point estimates of the slopes suggest that The Netherlands have experienced warming in the winter similar in magnitude to the global average but cooling in the late summer.

In Tables 10 to 13 we report additional tests of various hypotheses of interest. Table 10 confirms that the slopes are not all zero and Table 11 confirms that they are not all equal. Of course, given

\footnotetext{
${ }^{2}$ This contrasts with the case of annual global temperature data where the errors exhibit positive serial correlation. See Woodward and Gray (1993) or Fomby and Vogelsang (2000).

${ }^{3}$ See Bloomfield (1992), Woodward and Gray (1993), Zheng and Basher (1999) and Fomby and Vogelsang (2000) for point estimates.
} 
our simulation evidence, rejections using $W_{H A C}$ have to be viewed with some caution given the over-rejection problem. Table 12 reports tests of the null hypothesis that three consecutive months have zero slopes. In most cases, the tests suggest no warming or cooling except in the winter (December-February) where the point estimates indicate warming. Table 13 reports tests of the null hypothesis that three consecutive months have the same slopes. In most cases, the tests do not reject this null which is consistent with all slopes being zero. The exceptions are January-March, December-February and July-September. In all these cases we have months with large positive or negative slopes grouped with a month with a zero slope or slope of opposite sign.

Finally, Tables 14 to 16 test whether the winter months that indicate positive trends have equal trends. The tests are computed pairwise. The tests suggest that the point estimates for December and March are consistent with the same slope. But, there is some evidence that the January and December slopes are different.

Overall, our empirical results strongly suggest that changes in temperatures over the past 300 years in The Netherlands vary considerably across the months. There is evidence of warming in the winter, especially January, and cooling in the summer, especially September.

\section{Conclusions and Direction for Future Research}

In this paper we develop tests that can be used to test general linear hypotheses regarding the linear trend slope parameters of a vector of trend stationary time series. As an alternative to traditional Wald tests based on HAC robust standard errors, we recommend the use of a $F$-tests based on the approach of Kiefer et al. (2000) and Kiefer and Vogelsang (2001). One of the new tests has much better finite sample size with comparable but slightly lower to the HAC based Wald test.

We apply the tests to monthly temperature data for the Netherlands. We find that winters in the Netherlands are getting warmer at about the same rate as the entire earth, but summers are in fact experiencing slight cooling. Clearly, at least for the Netherlands, global warming is not having a uniform impact across the months or seasons.

Given that little research has been done on multivariate trend function inference, there are many directions to extend the approaches in this paper. The most obvious and simple extension would be to consider kernels other than the Bartlett kernel. More interesting extensions include models with unit root or near unit root errors, models with cointegrated errors, and models with higher order trends or trends with structural change. 


\section{References}

Andrews, D. W. K. (1991), Heteroskedasticity and Autocorrelation Consistent Covariance Matrix Estimation, Econometrica 59, 817-854.

Andrews, D. W. K. and Monahan, J. C. (1992), An Improved Heteroskedasticity and Autocorrelation Consistent Covariance Matrix Estimator, Econometrica 60, 953-966.

Bloomfield, P. (1992), Trends in Global Temperatures, Climate Change 21, 275-287.

Canjels, E. and Watson, M. W. (1997), Estimating Deterministic Trends in the Presence of Serially Correlated Errors, Review of Economics and Statistics May, 184-200.

Carlino, G. A. and Mills, L. O. (1993), Are U.S. Regional Incomes Converging?, Journal of Monetary Economics 32, 335-346.

Fomby, T. and Vogelsang, T. J. (2000), The Application of Size Robust Trend Analysis to Global Warming Temperature Series, mimeo, Department of Economics, Cornell University.

Grenander, U. and Rosenblatt, M. (1957), Statistical Analysis of Stationary Time Series, Wiley, New York.

Hobijn, B. and Franses, P. H. (2000), Asymptotically Perfect and Relative Convergence of Productivity, Journal of Applied Econometrics 15, 59-81.

Kiefer, N. M. and Vogelsang, T. J. (2000), A New Approach to the Asymptotics of Heteroskedasticity-Autocorrelation Robust Testing, mimeo, Department of Economics, Cornell University.

Kiefer, N. M. and Vogelsang, T. J. (2001), Heteroskedasticity-Autocorrelation Robust Standard Errors Using the Bartlett Kernel Without Truncation, mimeo, Department of Economics, Cornell University.

Kiefer, N. M., Vogelsang, T. J. and Bunzel, H. (2000), Simple Robust Testing of Regression Hypotheses, Econometrica 68, 695-714.

Loewy, M. B. and Papell, D. H. (1995), Are U.S. Regional Incomes Converging? Some Further Evidence, Journal of Monetary Economics 38, 587-598. 
Newey, W. K. and West, K. D. (1987), A Simple, Positive Semi-Definite, Heteroskedasticity and Autocorrelation Consistent Covariance Matrix, Econometrica 55, 703-708.

Park, J. Y. and Phillips, P. C. B. (1988), Regressions with Integrated Processes, Part I, Econometric Theory 4, 468-498.

Perron, P. (1991), A Test for Changes in a Polynomial Trend Function for a Dynamic Time Series, manuscript, Princeton University.

Phillips, P. C. B. and Durlauf, S. N. (1986), Multiple Regression with Integrated Processes, Review of Economic Studies 53, 473-496.

Tomljanovich, M. and Vogelsang, T. J. (2001), Are U.S. Regions Converging? Using New Econometric Methods to Examine Old Issues, Empirical Economics, forthcoming.

Vogelsang, T. J. (1997), Wald-Type Tests for Detecting Shifts in the Trend Function of a Dynamic Time Series, Econometric Theory 13, 818-849.

Vogelsang, T. J. (1998), Trend Function Hypothesis Testing in the Presence of Serial Correlation Correlation Parameters, Econometrica 65, 123-148.

Woodward, W. A. and Gray, H. L. (1993), Global Warming and the Problem of Testing for Trend in Time Series Data, Journal of Climate 6, 953-962.

Zheng, X. and Basher, R. E. (1999), Structural Time Series Models and Trend Detection in Global and Regional Temperature Series, Journal of Climate 12, 2347-2358. 


\section{Appendix}

This appendix contains the proofs of the various results derived in this paper.

Proofs of (12) and (17): Simple algebra along with (2) and (9) gives

$$
\begin{aligned}
T^{-\frac{1}{2}} \widehat{S}_{[r T]} & =T^{-\frac{1}{2}} \sum_{t=1}^{[r T]} \widehat{u}_{t} \\
& =T^{-\frac{1}{2}} \sum_{t=1}^{[r T]}\left(\widetilde{y}_{t}-\widehat{\beta} \widetilde{t}\right) \\
& =T^{-\frac{1}{2}} \sum_{t=1}^{[r T]}\left(\widehat{\beta t}+u_{t}-\bar{u}-\widehat{\beta} \widetilde{t}\right) \\
& =T^{-\frac{1}{2}} \sum_{t=1}^{[r T]}\left(u_{t}-\bar{u}\right)-T^{\frac{3}{2}}(\widehat{\beta}-\beta) T^{-2} \sum_{t=1}^{[r T]} \widetilde{t} \\
& \Rightarrow \Lambda\left[W_{n}(r)-r W_{n}(1)-12 V_{n}(1) \int_{0}^{r}\left(s-\frac{1}{2}\right) d s\right] \\
& =\Lambda \widehat{V}_{n}(r),
\end{aligned}
$$

which proves (12). Similarly, using (2), (5), (6) and (9) it follows that

$$
\begin{aligned}
T^{-\frac{3}{2}} \widetilde{S}_{[r T]} & =T^{-\frac{3}{2}} \sum_{t=1}^{[r T]} \widetilde{t} \widehat{u}_{t} \\
& =T^{-\frac{3}{2}} \sum_{t=1}^{[r T]} \widetilde{t}\left(\widetilde{y}_{t}-\widehat{\beta} \widetilde{t}\right) \\
& =T^{-\frac{3}{2}} \sum_{t=1}^{[r T]} \widetilde{t}\left(\beta \widetilde{t}+u_{t}-\bar{u}-\widehat{\beta} \widetilde{t}\right) \\
& =T^{-\frac{3}{2}} \sum_{t=1}^{[r T]} \widetilde{t}\left(u_{t}-\bar{u}\right)-T^{\frac{3}{2}}(\widehat{\beta}-\beta) T^{-3} \sum_{t=1}^{[r T]} \widetilde{t}^{2} \\
& \Rightarrow \Lambda\left[\int_{0}^{r}\left(s-\frac{1}{2}\right) d W_{n}(s)-\left(\int_{0}^{r}\left(s-\frac{1}{2}\right) d s\right) W_{n}(1)-12 V_{n}(1) \int_{0}^{r}\left(s-\frac{1}{2}\right)^{2} d s\right] \\
& =\Lambda \widetilde{V}_{n}(r),
\end{aligned}
$$

which proves (17).

Proof of Theorem 1: Simple algebra under $H_{0}$ gives

$$
F_{1}^{*}=T^{\frac{3}{2}}(R(\widehat{\beta}-\beta))^{\prime}\left[R\left(T^{-3} \sum_{t=1}^{T} \widetilde{t}^{2}\right)^{-1} T^{-2} \widetilde{\Omega}_{S_{T}=T}\left(T^{-3} \sum_{t=1}^{T} \widetilde{t}^{2}\right)^{-1} R^{\prime}\right]^{-1} T^{\frac{3}{2}}(R(\widehat{\beta}-\beta)) / q .
$$


Using (5), (9) and(13) it follows that

$$
F_{1}^{*} \Rightarrow\left[R \Lambda V_{n}(1)\right]^{\prime}\left[2 R \Lambda \int_{0}^{1} \widetilde{V}_{n}(r) \widetilde{V}_{n}(r)^{\prime} d r \Lambda^{\prime} R^{\prime}\right]^{-1}\left[R \Lambda V_{n}(1)\right] / q .
$$

We can write $R \Lambda W_{n}(r)$ as $\Lambda^{*} W_{q}(r)$, where $\Lambda^{*}$ is a $q \times q$ matrix with $\Lambda^{*} \Lambda^{*^{\prime}}=R \Lambda \Lambda^{\prime} R^{\prime}$ because $W_{n}(r)$ is a vector of independent Gaussian random variables. Therefore, direct algebra allows us to write $R \Lambda V_{n}(r)$ as $\Lambda^{*} V_{q}(r)$, and $R \Lambda \widetilde{V}_{n}(r)$ as $\Lambda^{*} \widetilde{V}_{q}(r)$. Using these representations gives

$$
\begin{aligned}
F_{1}^{*} & \Rightarrow\left[\Lambda^{*} V_{q}(1)\right]^{\prime}\left[2 \Lambda^{*} \int_{0}^{1} \widetilde{V}_{q}(r) \widetilde{V}_{q}(r)^{\prime} d r \Lambda^{*^{\prime}}\right]^{-1}\left[\Lambda^{*} V_{q}(1)\right] / q \\
& =V_{q}(1)^{\prime}\left[2 \int_{0}^{1} \widetilde{V}_{q}(r) \widetilde{V}_{q}(r)^{\prime} d r\right]^{-1} V_{q}(1) / q
\end{aligned}
$$

Using similar arguments as for $F_{1}^{*}$, it follows from (5), (9) and(18) that

$$
\begin{aligned}
F_{2}^{*} & =T^{\frac{3}{2}}[R(\widehat{\beta}-\beta)]^{\prime}\left[R\left(T^{-3} \sum_{t=1}^{T} \widetilde{t}^{2}\right)^{-1} \widehat{\Omega}_{S_{T}=T} R^{\prime}\right]^{-1} T^{\frac{3}{2}}[R(\widehat{\beta}-\beta)] / q \\
& \Rightarrow\left[R \Lambda V_{n}(1)\right]^{\prime}\left[\frac{1}{6} R \Lambda \int_{0}^{1} \widehat{V}_{n}(r) \widehat{V}_{n}(r)^{\prime} d r \Lambda^{\prime} R^{\prime}\right]^{-1}\left[R \Lambda V_{n}(1)\right] / q \\
& =V_{q}(1)^{\prime}\left[\frac{1}{6} \int_{0}^{1} \widehat{V}_{q}(r) \widehat{V}_{q}(r)^{\prime} d r\right]^{-1} V_{q}(1) / q
\end{aligned}
$$

Proof of Theorem 2: Under the local alternative we have

$$
\widehat{\beta}_{1}-\beta_{0}=c T^{-\frac{3}{2}}+\left(\sum_{t=1}^{T} \widetilde{t}^{2}\right)^{-1} \sum_{t=1}^{T} \widetilde{t} u_{t} .
$$

Therefore, using (5) and(6) it immediately follows that

$$
T^{\frac{3}{2}}\left(\widehat{\beta}_{1}-\beta_{0}\right)=c+\left(T^{-3} \sum_{t=1}^{T} \widetilde{t}^{2}\right)^{-1} T^{-3 / 2} \sum_{t=1}^{T} \widetilde{t} u_{t} \Rightarrow c+12 \sigma_{1} V_{1}(1)
$$

Simple algebra gives

$$
\begin{gathered}
t_{1}^{*}=\frac{T^{\frac{3}{2}}\left(\widehat{\beta}_{1}-\beta_{0}\right)}{\left[\left(T^{-3} \sum_{t=1}^{T} \widetilde{t}^{2}\right)^{-1} T^{-2} \widetilde{\Omega}_{S_{T}=T}\left(T^{-3} \sum_{t=1}^{T} \widetilde{t}^{2}\right)^{-1}\right]^{\frac{1}{2}}}, \\
t_{2}^{*}=\frac{T^{\frac{3}{2}}\left(\widehat{\beta}_{1}-\beta_{0}\right)}{\left[\left(T^{-3} \sum_{t=1}^{T} \widetilde{t}^{2}\right)^{-1} \widehat{\Omega}_{S_{T}=T}\right]^{\frac{1}{2}}},
\end{gathered}
$$


and

$$
t_{H A C}=\frac{T^{\frac{3}{2}}\left(\widehat{\beta}_{1}-\beta_{0}\right)}{\left[\left(T^{-3} \sum_{t=1}^{T} \widetilde{t}^{2}\right)^{-1} \widehat{\Omega}_{H A C}\right]^{\frac{1}{2}}} .
$$

Because $\widetilde{\Omega}_{S_{T}=T}, \widehat{\Omega}_{S_{T}=T}$ and $\widehat{\Omega}_{H A C}$ are exactly invariant to $\beta_{1}$, their limits are the same as under the null hypothesis. Therefore, using (22), we have

$$
\begin{gathered}
t_{1}^{*} \Rightarrow \frac{c+12 \sigma_{1} V_{1}(1)}{\left[288 \sigma_{1}^{2} \int_{0}^{1} \widetilde{V}_{1}(r)^{2} d r\right]^{\frac{1}{2}}}, \\
t_{2}^{*} \Rightarrow \frac{c+12 \sigma_{1} V_{1}(1)}{\left[24 \sigma_{1}^{2} \int_{0}^{1} \widehat{V}_{1}(r)^{2} d r\right]^{\frac{1}{2}}},
\end{gathered}
$$

and

$$
t_{H A C} \Rightarrow \frac{c+12 \sigma_{1} V_{1}(1)}{\left[12 \sigma_{1}^{2}\right]^{\frac{1}{2}}} .
$$

Simple algebra further completes the proof.

Table 1: Asymptotic critical values for $t_{1}^{*}$ and $t_{2}^{* 1}$

\begin{tabular}{ccccc}
\hline & 0.90 & 0.95 & 0.975 & 0.99 \\
\hline$t_{1}^{*}$ & 3.315 & 4.566 & 5.820 & 7.416 \\
$t_{2}^{*}$ & 3.898 & 5.222 & 6.482 & 8.100 \\
\hline
\end{tabular}

1 The tests concern a two-sided alternative hypothesis. 
Table 2: Asymptotic critical values of $F_{1}^{*}$ for $q$ restrictions

\begin{tabular}{ccccc}
\hline$q$ & 0.90 & 0.95 & 0.975 & 0.99 \\
\hline 1 & 20.81 & 33.63 & 48.42 & 72.23 \\
2 & 26.27 & 38.10 & 51.08 & 71.04 \\
3 & 30.97 & 42.38 & 54.66 & 73.40 \\
4 & 34.90 & 46.75 & 59.35 & 76.75 \\
5 & 38.63 & 49.82 & 61.88 & 78.29 \\
6 & 42.76 & 54.68 & 67.53 & 83.98 \\
7 & 47.29 & 59.32 & 71.77 & 88.54 \\
8 & 50.74 & 62.87 & 74.33 & 90.29 \\
9 & 54.63 & 67.17 & 80.14 & 95.76 \\
10 & 58.26 & 70.99 & 83.31 & 100.1 \\
11 & 61.82 & 74.51 & 87.45 & 103.3 \\
12 & 66.02 & 79.17 & 92.49 & 109.2 \\
13 & 69.26 & 82.45 & 95.61 & 113.1 \\
14 & 72.73 & 86.02 & 98.94 & 115.0 \\
15 & 75.98 & 88.70 & 102.5 & 119.8 \\
16 & 79.34 & 93.04 & 106.7 & 124.1 \\
17 & 82.98 & 96.55 & 110.4 & 127.1 \\
18 & 86.52 & 101.3 & 114.9 & 133.0 \\
19 & 90.36 & 105.5 & 119.6 & 138.4 \\
20 & 93.16 & 108.1 & 122.3 & 141.3 \\
21 & 96.39 & 111.1 & 125.1 & 144.3 \\
22 & 99.96 & 114.6 & 129.7 & 147.4 \\
23 & 103.1 & 117.9 & 133.2 & 150.9 \\
24 & 107.3 & 122.3 & 137.3 & 156.8 \\
25 & 110.4 & 125.9 & 140.9 & 160.7 \\
26 & 114.1 & 129.4 & 144.1 & 163.6 \\
27 & 117.4 & 133.5 & 148.8 & 167.9 \\
28 & 120.3 & 136.3 & 151.5 & 171.6 \\
29 & 123.6 & 139.6 & 155.7 & 175.5 \\
30 & 126.7 & 143.5 & 158.9 & 179.2 \\
\hline & & & & \\
& & & & \\
&
\end{tabular}


Table 3: Asymptotic critical values of $F_{2}^{*}$ for $q$ restrictions

\begin{tabular}{ccccc}
\hline$q$ & 0.90 & 0.95 & 0.975 & 0.99 \\
\hline 1 & 20.14 & 41.53 & 58.57 & 83.96 \\
2 & 28.90 & 40.68 & 53.58 & 73.50 \\
3 & 30.95 & 41.45 & 52.86 & 68.67 \\
4 & 33.26 & 43.84 & 54.60 & 69.30 \\
5 & 35.51 & 45.43 & 55.86 & 70.14 \\
6 & 38.26 & 48.39 & 58.91 & 73.36 \\
7 & 41.22 & 51.35 & 61.62 & 75.87 \\
8 & 43.50 & 53.25 & 63.18 & 76.71 \\
9 & 46.36 & 56.86 & 67.24 & 80.55 \\
10 & 49.05 & 58.90 & 68.92 & 93.06 \\
11 & 51.56 & 62.08 & 71.92 & 85.85 \\
12 & 54.54 & 65.01 & 74.95 & 88.65 \\
13 & 56.49 & 67.07 & 77.41 & 92.66 \\
14 & 59.43 & 69.98 & 80.32 & 93.61 \\
15 & 61.65 & 72.32 & 82.74 & 97.02 \\
16 & 64.33 & 74.83 & 85.63 & 99.79 \\
17 & 66.95 & 77.89 & 88.15 & 102.1 \\
18 & 69.69 & 80.75 & 91.93 & 106.0 \\
19 & 72.52 & 84.24 & 95.18 & 109.2 \\
20 & 74.35 & 86.17 & 97.20 & 112.2 \\
21 & 76.74 & 87.99 & 99.32 & 113.2 \\
22 & 79.45 & 91.12 & 102.6 & 116.1 \\
23 & 81.77 & 93.27 & 104.6 & 118.3 \\
24 & 84.53 & 96.56 & 108.5 & 123.7 \\
25 & 86.94 & 99.07 & 110.8 & 125.3 \\
26 & 89.57 & 102.0 & 113.7 & 128.6 \\
27 & 91.88 & 104.5 & 116.5 & 131.6 \\
28 & 94.40 & 106.8 & 119.2 & 134.2 \\
29 & 96.89 & 109.4 & 121.3 & 136.7 \\
30 & 99.41 & 111.8 & 124.2 & 140.0 \\
\hline & & & &
\end{tabular}


Table 4: Empirical size and size-corrected power of the three tests, without and with prewhitening. Testing for a single zero restriction on $\beta(q=1)^{1}$

\begin{tabular}{lllllccc}
\hline \multicolumn{1}{c}{} & \multicolumn{9}{c}{ No prewhitening } & \multicolumn{2}{c}{$\operatorname{VAR}(1)$ prewhitening } \\
$\rho$ & $\beta$ & $F_{1}^{*}$ & $F_{2}^{*}$ & $W_{H A C}$ & $F_{1}^{*}$ & $F_{2}^{*}$ & $W_{H A C}$ \\
\hline 0 & 0 & 0.047 & 0.043 & 0.061 & 0.039 & 0.035 & 0.064 \\
& 0.005 & 0.227 & 0.270 & 0.305 & 0.228 & 0.267 & 0.293 \\
& 0.010 & 0.622 & 0.743 & 0.804 & 0.629 & 0.736 & 0.792 \\
& 0.020 & 0.980 & 0.998 & 1.000 & 0.984 & 0.998 & 0.999 \\
0.2 & 0 & 0.052 & 0.048 & 0.086 & 0.039 & 0.037 & 0.066 \\
& 0.005 & 0.161 & 0.194 & 0.210 & 0.173 & 0.189 & 0.205 \\
& 0.010 & 0.465 & 0.548 & 0.618 & 0.473 & 0.544 & 0.608 \\
& 0.020 & 0.925 & 0.976 & 0.996 & 0.937 & 0.978 & 0.995 \\
0.4 & 0 & 0.058 & 0.054 & 0.106 & 0.040 & 0.036 & 0.069 \\
& 0.005 & 0.117 & 0.134 & 0.135 & 0.127 & 0.141 & 0.132 \\
& 0.010 & 0.298 & 0.359 & 0.396 & 0.310 & 0.364 & 0.387 \\
& 0.020 & 0.753 & 0.851 & 0.914 & 0.783 & 0.859 & 0.906 \\
0.6 & 0 & 0.063 & 0.062 & 0.134 & 0.040 & 0.036 & 0.080 \\
& 0.005 & 0.092 & 0.091 & 0.100 & 0.088 & 0.098 & 0.096 \\
& 0.010 & 0.173 & 0.194 & 0.223 & 0.178 & 0.205 & 0.216 \\
& 0.020 & 0.480 & 0.537 & 0.618 & 0.476 & 0.567 & 0.616 \\
0.8 & 0 & 0.088 & 0.097 & 0.192 & 0.041 & 0.038 & 0.098 \\
& 0.005 & 0.068 & 0.071 & 0.062 & 0.065 & 0.064 & 0.068 \\
& 0.010 & 0.093 & 0.106 & 0.102 & 0.090 & 0.106 & 0.103 \\
& 0.020 & 0.184 & 0.212 & 0.232 & 0.193 & 0.215 & 0.231 \\
& & & & & & & \\
\hline
\end{tabular}

The cells contain the finite sample empirical rejection probabilities (at a $5 \%$ significance level), where the power is corrected for the empirical size. The number of replications is 2000, and the sample size is 100 . All series have $\operatorname{AR}(1)$ errors with parameter $\rho$. The Wald test is implemented using a Bartlett kernel with Andrews VAR(1) data-dependent bandwith.

1 The critical values for the $F_{1}^{*}$ and $F_{2}^{*}$ tests can be found in Table 2 and Table 3 for $q=1$, respectively. 
Table 5: Empirical size and size-corrected power of the three tests, without and with prewhitening. Testing for two zero restrictions on $\beta(q=2)^{1}$

\begin{tabular}{|c|c|c|c|c|c|c|c|}
\hline \multirow[b]{2}{*}{$\rho$} & \multirow[b]{2}{*}{$\beta$} & \multicolumn{3}{|c|}{ No prewhitening } & \multicolumn{3}{|c|}{$\operatorname{VAR}(1)$ prewhitening } \\
\hline & & $F_{1}^{*}$ & $F_{2}^{*}$ & $W_{H A C}$ & $F_{1}^{*}$ & $F_{2}^{*}$ & $W_{H A C}$ \\
\hline \multirow[t]{4}{*}{0} & 0 & 0.050 & 0.049 & 0.070 & 0.036 & 0.037 & 0.074 \\
\hline & 0.005 & 0.294 & 0.335 & 0.411 & 0.310 & 0.348 & 0.401 \\
\hline & 0.010 & 0.773 & 0.856 & 0.958 & 0.805 & 0.868 & 0.954 \\
\hline & 0.020 & 0.996 & 1.000 & 1.000 & 0.995 & 1.000 & 1.000 \\
\hline \multirow[t]{4}{*}{0.2} & 0 & 0.058 & 0.060 & 0.107 & 0.038 & 0.040 & 0.081 \\
\hline & 0.005 & 0.209 & 0.222 & 0.285 & 0.217 & 0.240 & 0.278 \\
\hline & 0.010 & 0.615 & 0.679 & 0.829 & 0.646 & 0.697 & 0.822 \\
\hline & 0.020 & 0.960 & 0.994 & 1.000 & 0.975 & 0.997 & 1.000 \\
\hline \multirow[t]{4}{*}{0.4} & 0 & 0.068 & 0.068 & 0.138 & 0.039 & 0.040 & 0.091 \\
\hline & 0.005 & 0.136 & 0.139 & 0.169 & 0.146 & 0.146 & 0.172 \\
\hline & 0.010 & 0.389 & 0.435 & 0.535 & 0.426 & 0.458 & 0.541 \\
\hline & 0.020 & 0.871 & 0.934 & 0.990 & 0.895 & 0.950 & 0.990 \\
\hline \multirow[t]{4}{*}{0.6} & 0 & 0.082 & 0.088 & 0.198 & 0.045 & 0.045 & 0.112 \\
\hline & 0.005 & 0.083 & 0.086 & 0.094 & 0.087 & 0.094 & 0.103 \\
\hline & 0.010 & 0.204 & 0.214 & 0.268 & 0.218 & 0.240 & 0.272 \\
\hline & 0.020 & 0.569 & 0.657 & 0.785 & 0.621 & 0.695 & 0.782 \\
\hline \multirow[t]{4}{*}{0.8} & 0 & 0.129 & 0.144 & 0.330 & 0.063 & 0.055 & 0.181 \\
\hline & 0.005 & 0.056 & 0.060 & 0.059 & 0.064 & 0.063 & 0.068 \\
\hline & 0.010 & 0.085 & 0.089 & 0.094 & 0.094 & 0.096 & 0.096 \\
\hline & 0.020 & 0.202 & 0.221 & 0.245 & 0.231 & 0.251 & 0.260 \\
\hline
\end{tabular}

The cells contain the finite sample empirical rejection probabilities (at a $5 \%$ significance level), where the power is corrected for the empirical size. The number of replications is 2000, and the sample size is 100 . All series have $\operatorname{AR}(1)$ errors with parameter $\rho$. The Wald test is implemented using a Bartlett kernel with Andrews VAR(1) data-dependent bandwith.

1 The critical values for the $F_{1}^{*}$ and $F_{2}^{*}$ tests can be found in Table 2 and Table 3 for $q=2$, respectively. 
Table 6: Empirical size and size-corrected power of the three tests, without and with prewhitening. Testing for three zero restrictions on $\beta(q=3)^{1}$

\begin{tabular}{lllllccc}
\hline \multicolumn{1}{c}{} & \multicolumn{9}{c}{ No prewhitening } & \multicolumn{2}{c}{$\operatorname{VAR}(1)$ prewhitening } \\
$\rho$ & $\beta$ & $F_{1}^{*}$ & $F_{2}^{*}$ & $W_{H A C}$ & $F_{1}^{*}$ & $F_{2}^{*}$ & $W_{H A C}$ \\
\hline 0 & 0 & 0.049 & 0.049 & 0.073 & 0.042 & 0.042 & 0.095 \\
& 0.005 & 0.333 & 0.379 & 0.488 & 0.329 & 0.373 & 0.477 \\
& 0.010 & 0.847 & 0.916 & 0.987 & 0.840 & 0.914 & 0.985 \\
& 0.020 & 0.996 & 1.000 & 1.000 & 0.997 & 1.000 & 1.000 \\
0.2 & 0 & 0.063 & 0.061 & 0.127 & 0.042 & 0.042 & 0.102 \\
& 0.005 & 0.232 & 0.253 & 0.332 & 0.226 & 0.246 & 0.312 \\
& 0.010 & 0.681 & 0.757 & 0.906 & 0.681 & 0.759 & 0.887 \\
& 0.020 & 0.982 & 0.997 & 1.000 & 0.985 & 0.998 & 1.000 \\
0.4 & 0 & 0.077 & 0.074 & 0.195 & 0.049 & 0.046 & 0.123 \\
& 0.005 & 0.154 & 0.173 & 0.197 & 0.149 & 0.165 & 0.197 \\
& 0.010 & 0.452 & 0.520 & 0.632 & 0.443 & 0.512 & 0.618 \\
& 0.020 & 0.916 & 0.965 & 0.999 & 0.912 & 0.970 & 0.997 \\
0.6 & 0 & 0.108 & 0.108 & 0.286 & 0.061 & 0.050 & 0.164 \\
& 0.005 & 0.088 & 0.108 & 0.111 & 0.085 & 0.104 & 0.101 \\
& 0.010 & 0.229 & 0.265 & 0.307 & 0.218 & 0.254 & 0.294 \\
& 0.020 & 0.656 & 0.746 & 0.855 & 0.647 & 0.752 & 0.851 \\
0.8 & 0 & 0.188 & 0.204 & 0.464 & 0.101 & 0.075 & 0.273 \\
& 0.005 & 0.056 & 0.058 & 0.061 & 0.061 & 0.062 & 0.067 \\
& 0.010 & 0.091 & 0.107 & 0.101 & 0.097 & 0.109 & 0.112 \\
& 0.020 & 0.242 & 0.284 & 0.281 & 0.251 & 0.282 & 0.312 \\
& & & & & & & \\
\hline
\end{tabular}

The cells contain the finite sample empirical rejection probabilities (at a $5 \%$ significance level), where the power is corrected for the empirical size. The number of replications is 2000, and the sample size is 100 . All series have $\operatorname{AR}(1)$ errors with parameter $\rho$. The Wald test is implemented using a Bartlett kernel with Andrews VAR(1) data-dependent bandwith.

1 The critical values for the $F_{1}^{*}$ and $F_{2}^{*}$ tests can be found in Table 2 and Table 3 for $q=3$, respectively. 
Table 7: Empirical size and size-corrected power of the three tests, without and with prewhitening. Testing for eleven zero restrictions on $\beta(q=11)^{1}$

\begin{tabular}{|c|c|c|c|c|c|c|c|}
\hline \multirow[b]{2}{*}{$\rho$} & \multirow[b]{2}{*}{$\beta$} & \multicolumn{3}{|c|}{ No prewhitening } & \multicolumn{3}{|c|}{$\operatorname{VAR}(1)$ prewhitening } \\
\hline & & $F_{1}^{*}$ & $F_{2}^{*}$ & $W_{H A C}$ & $F_{1}^{*}$ & $F_{2}^{*}$ & $W_{H A C}$ \\
\hline \multirow[t]{4}{*}{0} & 0 & 0.066 & 0.059 & 0.158 & 0.121 & 0.070 & 0.322 \\
\hline & 0.005 & 0.655 & 0.684 & 0.856 & 0.577 & 0.629 & 0.789 \\
\hline & 0.010 & 0.993 & 0.995 & 1.000 & 0.984 & 0.994 & 1.000 \\
\hline & 0.020 & 1.000 & 1.000 & 1.000 & 0.999 & 1.000 & 1.000 \\
\hline \multirow[t]{4}{*}{0.2} & 0 & 0.128 & 0.116 & 0.419 & 0.168 & 0.099 & 0.408 \\
\hline & 0.005 & 0.435 & 0.448 & 0.560 & 0.359 & 0.426 & 0.535 \\
\hline & 0.010 & 0.950 & 0.966 & 0.998 & 0.914 & 0.953 & 0.996 \\
\hline & 0.020 & 1.000 & 1.000 & 1.000 & 0.999 & 1.000 & 1.000 \\
\hline \multirow[t]{4}{*}{0.4} & 0 & 0.266 & 0.221 & 0.702 & 0.273 & 0.150 & 0.543 \\
\hline & 0.005 & 0.239 & 0.258 & 0.254 & 0.208 & 0.226 & 0.281 \\
\hline & 0.010 & 0.798 & 0.805 & 0.860 & 0.717 & 0.748 & 0.886 \\
\hline & 0.020 & 0.997 & 0.999 & 1.000 & 0.993 & 0.997 & 1.000 \\
\hline \multirow[t]{4}{*}{0.6} & 0 & 0.504 & 0.460 & 0.920 & 0.490 & 0.311 & 0.750 \\
\hline & 0.005 & 0.123 & 0.135 & 0.122 & 0.114 & 0.109 & 0.126 \\
\hline & 0.010 & 0.421 & 0.429 & 0.386 & 0.364 & 0.375 & 0.472 \\
\hline & 0.020 & 0.929 & 0.948 & 0.936 & 0.891 & 0.913 & 0.980 \\
\hline \multirow[t]{4}{*}{0.8} & 0 & 0.849 & 0.824 & 0.999 & 0.841 & 0.705 & 0.956 \\
\hline & 0.005 & 0.073 & 0.067 & 0.065 & 0.067 & 0.066 & 0.067 \\
\hline & 0.010 & 0.142 & 0.141 & 0.137 & 0.140 & 0.124 & 0.142 \\
\hline & 0.020 & 0.495 & 0.464 & 0.447 & 0.419 & 0.431 & 0.482 \\
\hline
\end{tabular}

The cells contain the finite sample empirical rejection probabilities (at a $5 \%$ significance level), where the power is corrected for the empirical size. The number of replications is 2000, and the sample size is 100 . All series have $\operatorname{AR}(1)$ errors with parameter $\rho$. The Wald test is implemented using a Bartlett kernel with Andrews VAR(1) data-dependent bandwith.

1 The critical values for the $F_{1}^{*}$ and $F_{2}^{*}$ tests can be found in Table 2 and Table 3 for $q=11$, respectively. 
Table 8: Empirical size of the three tests, without and with prewhitening. Testing for eleven zero restrictions on $\beta(q=11)$ for 288 observations $^{1}$

\begin{tabular}{lcccccr}
\hline \multicolumn{4}{c}{ No prewhitening } & \multicolumn{2}{c}{$\operatorname{VAR}(1)$ prewhitening } \\
$\rho$ & $F_{1}^{*}$ & $F_{2}^{*}$ & $W_{H A C}$ & $F_{1}^{*}$ & $F_{2}^{*}$ & $W_{H A C}$ \\
\hline 0 & 0.045 & 0.047 & 0.079 & 0.040 & 0.038 & 0.116 \\
0.2 & 0.074 & 0.068 & 0.199 & 0.049 & 0.040 & 0.138 \\
0.4 & 0.118 & 0.098 & 0.324 & 0.063 & 0.047 & 0.180 \\
0.6 & 0.193 & 0.162 & 0.529 & 0.099 & 0.064 & 0.272 \\
0.8 & 0.431 & 0.373 & 0.850 & 0.260 & 0.154 & 0.532 \\
\hline
\end{tabular}

The cells contain the finite sample empirical rejection probabilities (at a $5 \%$ significance level). The number of replications is 2000, and the sample size is 288. All series have $\operatorname{AR}(1)$ errors with parameter $\rho$. The Wald test is implemented using a Bartlett kernel with Andrews VAR(1) data-dependent bandwith.

1 The critical values for the $F_{1}^{*}$ and $F_{2}^{*}$ tests can be found in Table 2 and Table 3 for $q=11$, respectively.

Table 9: Single equation estimation results for monthly temperatures in The Netherlands. ${ }^{1}$

\begin{tabular}{|c|c|c|c|c|c|c|c|c|c|}
\hline \multirow[b]{2}{*}{ Month } & \multirow[b]{2}{*}{$\widehat{\beta}$} & \multirow[b]{2}{*}{$\widehat{\rho}_{1}$} & \multirow[b]{2}{*}{ pval } & \multicolumn{3}{|c|}{ No prewhitening } & \multicolumn{3}{|c|}{ VAR(1) prewhitening } \\
\hline & & & & $t_{1}^{*}$ & $t_{2}^{*}$ & $t_{H A C}$ & $t_{1}^{*}$ & $t_{2}^{*}$ & $t_{H A C}$ \\
\hline January & 0.0069 & -0.049 & 0.812 & $5.3279^{*}$ & $7.3991^{*}$ & * $3.7998^{* * *}$ & ** $5.4271^{*}$ & $7.3771^{* *}$ & ** $3.9302^{* *}$ \\
\hline February & -0.0004 & -0.048 & 0.209 & -1.0673 & -1.0871 & $-0.2651-$ & -1.0329 & -1.0733 & -0.2756 \\
\hline March & 0.0038 & 0.086 & 0.313 & 3.6930 & 6.2973 & $2.8415^{* * *}$ & k* 4.0011 & $6.1929^{*}$ & ${ }^{*} 2.6925^{* * *}$ \\
\hline April & 0.0003 & 0.094 & 0.077 & 0.5562 & 0.8601 & 0.3108 & 0.5775 & 0.8497 & 0.2894 \\
\hline May & 0.0011 & 0.030 & 0.529 & 2.8487 & 3.5735 & 1.1132 & 3.7023 & 3.4118 & 1.1369 \\
\hline June & 0.0005 & -0.016 & 0.250 & 1.5635 & 1.3860 & 0.5508 & 1.6306 & 1.3589 & 0.5613 \\
\hline July & 0.0006 & 0.017 & 0.821 & 2.0568 & 2.4954 & 0.6893 & 2.3626 & 2.4767 & 0.6770 \\
\hline August & -0.0004 & 0.118 & 0.298 & -0.9808 & -1.2882 & -0.4830 & -1.0523 & -1.2816 & -0.4521 \\
\hline September & -0.0019 & -0.038 & 0.990 & -4.0678 & $-5.6466^{*}$ & $-2.3998^{* *}$ & *ㅡ 3.8418 & $-5.6399 *$ & ${ }^{*}-2.4126^{* * *}$ \\
\hline October & 0.0004 & 0.136 & 0.278 & 0.5327 & 0.8221 & 0.4181 & 0.5657 & 0.8168 & 0.3880 \\
\hline November & 0.0006 & 0.083 & 0.190 & 0.3967 & 0.6886 & 0.5507 & 0.3965 & 0.6850 & 0.5375 \\
\hline December & 0.0028 & 0.003 & 0.994 & 1.8537 & 3.1072 & $1.7254^{*}$ & 1.8718 & 3.1037 & $1.7397^{*}$ \\
\hline
\end{tabular}


Table 10: Joint tests for the restriction that all months have slope equal to zero. ${ }^{1}$

\begin{tabular}{lrc} 
Test statistic & No prewhitening & VAR $(1)$ prewhitening \\
\hline$F_{1}^{*}$ & $123.8^{* * *}$ & $135.2^{* * *}$ \\
$F_{2}^{*}$ & $72.52^{* *}$ & $70.84^{* *}$ \\
$W_{H A C}$ & $41.74^{* * *}$ & $46.77^{* * *}$ \\
\hline
\end{tabular}

*** Significant at the 0.01 level, ${ }^{* *}$ at the 0.05 level

1 The critical values for the $F_{1}^{*}$ and $F_{2}^{*}$ tests can be found in Table 2 and Table 3 for $q=12$, respectively.

Table 11: Joint tests for the restriction that all months have the same slope ${ }^{1}$

\begin{tabular}{lrc}
\hline & & \\
Test statistic & No prewhitening & VAR $(1)$ prewhitening \\
\hline$F_{1}^{*}$ & $95.14^{* *}$ & $86.79^{* *}$ \\
$F_{2}^{*}$ & $67.84^{* *}$ & 59.09 \\
$W_{H A C}$ & $41.43^{* * *}$ & $46.70^{* * *}$ \\
\hline \multicolumn{2}{c}{$* *$ Significant at the 0.01 level, ${ }^{* *}$ at the 0.05 level } \\
1 The critical values for the $F_{1}^{*}$ and $F_{2}^{*}$ tests can be found in Table \\
2 and Table 3 for $q=11$, respectively.
\end{tabular}

Table 12: Joint tests that the trend slopes in three sequential months are equal to zero. ${ }^{1}$

\begin{tabular}{lcccccc}
\hline & \multicolumn{4}{c}{ No prewhitening } & \multicolumn{3}{c}{ VAR(1) prewhitening } \\
Months & $F_{1}^{*}$ & $F_{2}^{*}$ & $W_{H A C}$ & $F_{1}^{*}$ & $F_{2}^{*}$ & $W_{H A C}$ \\
\hline Jan.-Mar. & 31.36 & 39.69 & $27.18^{* * *}$ & 24.52 & 38.97 & $28.27^{* * *}$ \\
Feb.-Apr. & 14.45 & 22.61 & $11.12^{* *}$ & 15.31 & 22.35 & $11.11^{* * *}$ \\
Mar.-May & 10.07 & 18.72 & $9.388^{* *}$ & 13.68 & 17.99 & $9.136^{* *}$ \\
Apr.-Jun. & 7.960 & 7.230 & 1.427 & 10.18 & 6.030 & 1.513 \\
May-Jul. & 2.926 & 6.874 & 1.716 & 5.389 & 6.042 & 1.842 \\
Jun.-Aug. & 8.785 & 7.430 & 1.425 & 8.942 & 7.366 & 1.509 \\
Jul.-Sep. & 32.72 & 39.15 & 6.817 & 22.50 & 36.84 & 7.404 \\
Aug.-Oct. & 34.27 & 33.05 & 6.712 & 28.81 & 30.74 & 6.861 \\
Sep.-Nov. & 29.02 & 30.01 & 7.245 & 28.85 & 29.18 & 7.364 \\
Oct.-Dec. & 6.281 & 9.052 & 3.087 & 6.014 & 8.816 & 3.145 \\
Nov.-Jan. & 37.03 & 33.48 & $16.52^{* * *}$ & 35.35 & 32.97 & $16.41^{* * *}$ \\
Dec.-Feb. & $74.90^{* * *}$ & $64.99^{* *}$ & $19.72^{* * *}$ & $71.08^{* * *}$ & $64.44^{* *}$ & $20.07^{* * *}$ \\
\hline
\end{tabular}

$* * *$ Significant at the 0.01 level, $* *$ at the 0.05 level

1 The critical values for the $F_{1}^{*}$ and $F_{2}^{*}$ tests can be found in Table 2 and Table 3 for $q=3$, respectively. 
Table 13: Joint tests for the equality of trend slopes in three sequential months. ${ }^{1}$

\begin{tabular}{lcccccc}
\hline \multicolumn{4}{c}{ No prewhitening } & \multicolumn{3}{c}{ VAR(1) prewhitening } \\
Months & $F_{1}^{*}$ & $F_{2}^{*}$ & $W_{H A C}$ & $F_{1}^{*}$ & $F_{2}^{*}$ & $W_{H A C}$ \\
\hline Jan.-Mar. & 26.44 & $51.22^{* *}$ & $15.75^{* * *}$ & 25.24 & $51.12^{* *}$ & $17.36^{* * *}$ \\
Feb.-Apr. & 14.72 & 33.27 & $10.12^{* * *}$ & 17.25 & 32.77 & $10.75^{* * *}$ \\
Mar.-May & 15.00 & 26.44 & 6.244 & 17.73 & 25.74 & $6.516^{* *}$ \\
Apr.-Jun. & 2.690 & 2.019 & 0.394 & 2.472 & 1.934 & 0.381 \\
May-Jul. & 1.385 & 0.847 & 0.262 & 1.583 & 0.829 & 0.250 \\
Jun.-Aug. & 9.862 & 10.25 & 1.318 & 9.258 & 10.13 & 1.356 \\
Jul.-Sep. & $42.88^{* *}$ & $58.17^{* *}$ & 5.103 & 26.26 & $54.92^{* *}$ & 5.521 \\
Aug.-Oct. & 35.42 & 38.54 & 4.811 & 35.09 & 35.25 & 4.575 \\
Sep.-Nov. & 16.06 & 19.65 & 5.603 & 17.25 & 18.87 & 5.258 \\
Oct.-Dec. & 9.120 & 13.11 & 1.866 & 8.744 & 12.79 & 1.891 \\
Nov.-Jan. & 31.84 & 39.54 & $8.233^{* *}$ & 29.24 & 38.89 & $8.683^{* *}$ \\
Dec.-Feb. & $62.97^{* *}$ & $61.69^{* *}$ & $13.96^{* * *}$ & $64.31^{* *}$ & $61.73^{* *}$ & $15.05^{* * *}$ \\
\hline
\end{tabular}

*** Significant at the 0.01 level, $* *$ at the 0.05 level

1 The critical values for the $F_{1}^{*}$ and $F_{2}^{*}$ tests can be found in Table 2 and Table 3 for $q=2$, respectively.

Table 14: Pairwise two-sided $t_{1}^{*}$ tests for equality of trend parameters for three months. Below (above) the diagonal are the results without (with) $\operatorname{VAR}(1$ ) prewhitening. ${ }^{1}$

\begin{tabular}{lccc} 
Month & January & March & December \\
\hline January & n.a. & 5.412 & $6.384^{* *}$ \\
March & 5.683 & n.a. & 1.340 \\
December & $6.488^{* *}$ & 1.507 & n.a. \\
\hline
\end{tabular}

*** Significant at the 0.01 level, ${ }^{* *}$ at the 0.05 level.

1 The sample runs from January 1706 to September 1993. The relevant critical values appear in Table 1. 
Table 15: Pairwise two-sided $t_{2}^{*}$ tests for equality of trend parameters for three months. Below (above) the diagonal are the results without (with) $\operatorname{VAR}(1)$ prewhitening. ${ }^{1}$

\begin{tabular}{lccr} 
Month & January & March & December \\
\hline January & n.a. & 5.330 & 6.437 \\
March & 5.400 & n.a. & 2.207 \\
December & 6.402 & 2.051 & n.a. \\
\hline
\end{tabular}

$* * *$ Significant at the 0.01 level, ${ }^{* *}$ at the 0.05 level.

1 The sample runs from January 1706 to September 1993. The relevant critical values appear in Table 1.

Table 16: Pairwise two-sided $t_{H A C}$ tests for equality of trend parameters for three months. Below (above) the diagonal are the results without (with) $\operatorname{VAR}(1)$ prewhitening. ${ }^{1}$

\begin{tabular}{lccr} 
Month & January & March & December \\
\hline January & n.a. & 1.527 & 1.933 \\
March & 1.529 & n.a. & 0.512 \\
December & 1.751 & 0.481 & n.a. \\
\hline
\end{tabular}

*** Significant at the 0.01 level, ${ }^{* *}$ at the 0.05 level.

1 The sample runs from January 1706 to September 1993. The relevant critical values appear in Table 1. 


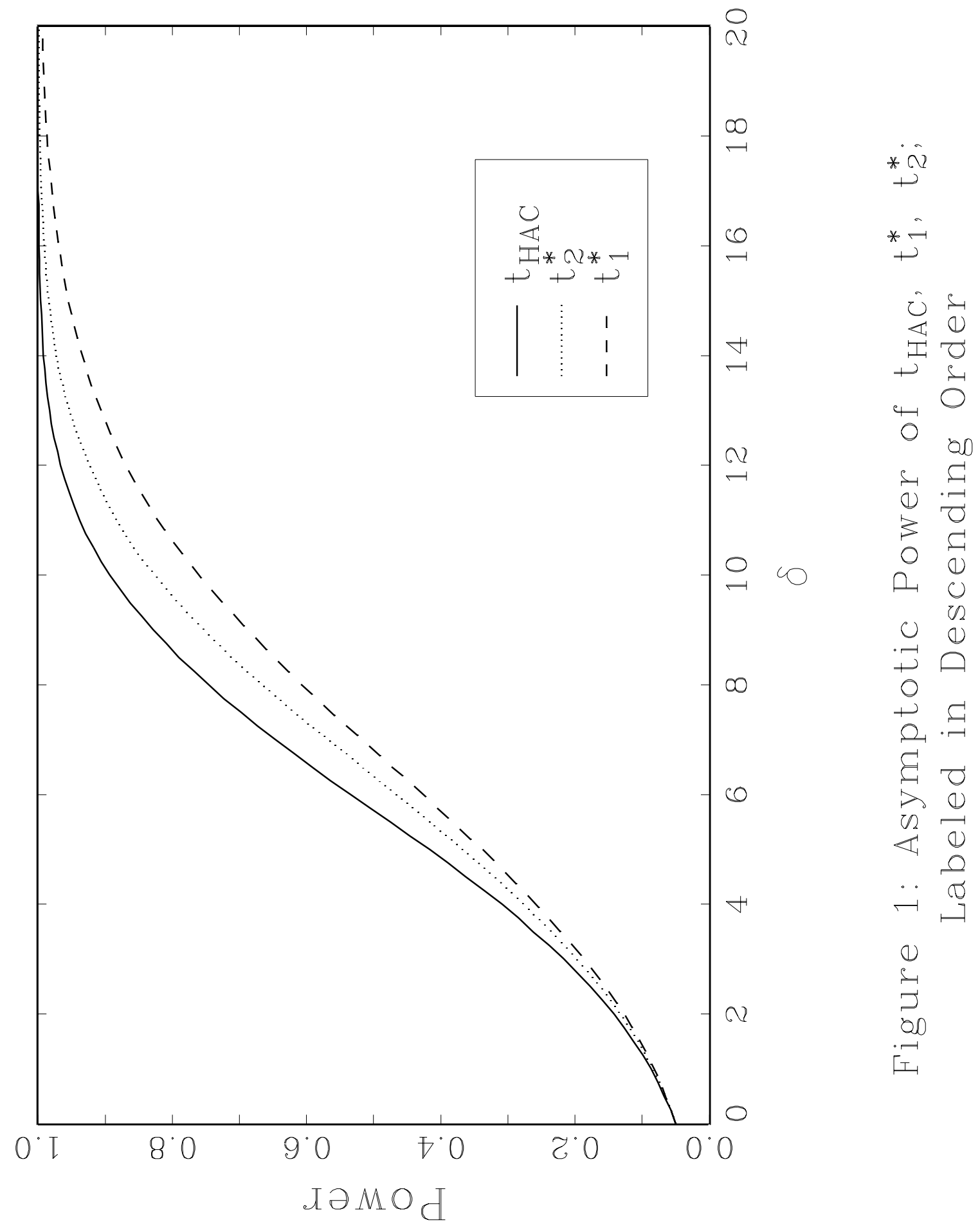

